\section{Implementing a systematic process for rapidly embedding sustainability within chemical engineering education: A case study of James Cook University, Australia}

\author{
Madoc Sheehan ${ }^{\mathrm{a}}$, Phil Schneider ${ }^{\mathrm{a}}$ and Cheryl \\ Desha $^{\text {b }}$
}

\section{Keywords}

${ }_{10}$ Sustainability, education, curriculum development, chemical engineering

\begin{abstract}
Sustainability has emerged as a primary context for engineering education in the 21st Century, particularly the sub-discipline of

15 chemical engineering. However, there is confusion over how to go about integrating sustainability knowledge and skills systemically within bachelor degrees. This paper addresses this challenge, using a case study of an Australian chemical engineering degree to highlight important practical

20 considerations for embedding sustainability at the core of the curriculum. The paper begins with context for considering a systematic process for rapid curriculum renewal. The authors then summarise a 2-year federally funded project, which comprised piloting a model for rapid curriculum renewal led by 25 the chemical engineering staff. Model elements contributing to the renewal of this engineering degree and described in this paper include: industry outreach; staff professional development; attribute identification and alignment; program mapping; and curriculum and teaching resource development.

30 Personal reflections on the progress and process of rapid curriculum renewal in sustainability by the authors and participating engineering staff will be presented as a means to discuss and identify methodological improvements, as well as highlight barriers to project implementation. It is hoped that this 35 paper will provide an example of a formalised methodology on which program reform and curriculum renewal for sustainability can be built upon in other higher education institutions.
\end{abstract}

\subsection{Introduction}

${ }_{40}$ Over the last two decades in particular, sustainability has emerged as a core context for $21^{\text {st }}$ Century living, to maintain or improve our quality of life without compromising the ability of future generations to meet their own needs (World commission on Environment and Development, 1987). Moreover, it is also 45 increasingly evident that sustainable development is only possible through a whole of system approach that both decouples economic growth from negative environmental pressures, and re- couples restorative processes (Smith et al., 2010).

The engineering profession is already playing a key role in this 50 transition to sustainable development, where everything from conceptual design through to operation, maintenance and decommissioning must embed sustainability (The Royal Academy of Engineering, 2007). Public statements by worldleading engineers across a range of disciplines echo this 55 imperative. For example, Andrew Ives concluded in the $120^{\text {th }}$ Presidential address to the Institution of Mechanical Engineers in 2004 that, "If humanity is to somehow manage to adjust its behaviour in any controlled way to a position of sustainability, we have limited time in which to do it...."The role of engineers is 60 to innovate in the quest for sustainable products and processes which will contain, limit or even reverse the ecological footprint of humanity in the 21st century."

Such statements within the engineering profession are reinforced in engineering education literature as summarised by Desha and 65 Hargroves (2009), where there is now broad acceptance of both the need to integrate sustainability into engineering education and the limited timeframe in which to transition to a state of education for sustainable development. The need and the limited timeframe provides the impetus for what Desha and Hargroves 70 refer to as "Rapid Curriculum Renewal" or RCR. In effect, an operational understanding of sustainability is rapidly becoming an essential tool that must be developed in engineering graduates. Indeed, concepts of sustainability and its practice must be integrated into engineering curricula so that the knowledge and 75 attitudes become second nature to the graduate's practice. Essentially, sustainability needs to become the context of engineering practice (Mulder et al., 2011).

Despite the identified need for the transition, chemical engineering academics such as Azapagic et al (2005), Davidson 80 et al ((2007), Byrne and Fitzpatrick (2009) and Byrne et al (2010) commonly conclude that most programmes have made only minor progress - if any - in increasing the exposure of students to sustainability issues, let alone embed sustainability as a core context for engineering studies.

85 Accompanying a need to reform existing curricula to incorporate sustainability is the aligned requirement that professional degrees, such as engineering, be accredited. Accrediting organisations for engineering programs in Australia, such as Engineers Australia (EA) and the Institution of Chemical Engineers (IChemE), have 90 recognised these developments and recently made significant changes to their accreditation documentation to reflect this new paradigm. For example, EA provides a list of graduate attributes or competencies that are aligned with a sustainability-informed ethos. These include (EA Stage 1 Competency Standards for ${ }_{95}$ Professional Engineers, Engineers Australia Accreditation Board (2006)): 
Table 1. Engineers Australia (EA) graduate 25 attributes/competencies aligned with sustainability (2006).

PE2.2 Understanding of social, cultural, global, and environmental responsibilities and the need to employ principles of sustainable development

- Appreciation of the interactions between technical systems and the social, cultural, environmental, economic and political context in which they operate, and the relationships between these factors.

- Appreciation of the imperatives of safety and of sustainability, and approaches to developing and maintaining safe and sustainable systems

- Ability to interact with people in other disciplines and professions to broaden knowledge, achieve multidisciplinary outcomes, and ensure that the engineering contribution is properly integrated into the total project.

- Appreciation of the nature of risk, both of a technical kind and in relation to

clients, users, the community and the environment

PE3.4 Understanding of professional and ethical responsibilities, and

commitment to them

5 The IChemE has played a leading role within the chemical engineering profession particularly in their recognition of the relevance of sustainability in chemical engineering education. In their guide for institutions seeking accreditation they take a different but complementary approach to EA, assessing

10 programmes against the "Learning Outcomes" achieved by students, often demonstrated in capstone learning experiences such as chemical process design. Core competencies relating to sustainability that are described in IChemE accreditation guide documentation (Institution of Chemical Engineers, 2006) are ${ }_{15}$ provided in Table 2.

50

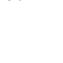

Table 2. Institute of Chemical Engineers (IChemE) graduate attributes/competencies aligned with sustainability.

\begin{tabular}{|l|}
\hline Understanding of the requirement for chemical engineering activities to promote \\
sustainable development. (Bachelor level) \\
\hline Understanding of the need for a high level of professional and ethical conduct in \\
chemical engineering. (Bachelor level) \\
\hline $\begin{array}{l}\text { Extensive knowledge and understanding of management and business practices, and } \\
\text { their limitations, and how these may be applied appropriately. (Masters level) }\end{array}$ \\
\hline $\begin{array}{l}\text { It is expected that courses throughout a programme include, illustrate and reinforce aspects of sustainability, safety, health al } \\
\text { environment (SHE) and, where possible ethics. (Bachelor level) }\end{array}$ \\
\hline
\end{tabular}


In 2008, IChemE also created a technology roadmap (Institution of Chemical Engineers, 2008) that clearly defines the institution's position on key short, medium and long-term issues affecting the profession. For example, in the report Australian members 5 identified energy and water as the two themes most relevant to the Australian chemical engineering community. Other key roadmap themes related to sustainability are highlighted in Table 3.

10 Table 3. Institute of Chemical Engineers (IChemE) technology roadmap statements aligned with sustainability.

Backs sustainable energy and supports the rapid pursuit of the global use of non-fossil primary energy sources

Is committed to driving the 3R's (Reduce, Reuse, Recycle)

mentality deeper into industry and consumers

Is supporting the introduction of innovative and sustainable technology throughout industry.

The main objective of this work is to outline a formal methodology for embedding sustainability into chemical engineering degree programs and to demonstrate its application via a case study degree program at James Cook 20 University in Australia. Particular emphasis is placed on deriving generic attributes for sustainability and mapping these attributes onto degree programs/subjects.

\section{Methodology Considerations for Curriculum Renewal}

25 Whilst engineering leaders and accreditation bodies have started to disseminate, formulate and recognise the importance of sustainability in engineering, accreditation documents and available literature lack a specific methodological approach to effectively guide and inform 30 higher education institutions in achieving comprehensive program reform. In the USA for example, a survey of 1368 engineering departments found curriculum development in sustainability to lack structure and organisation (Davidson et al., 2007). In Australia a 2007 report to the Learning and Teaching

${ }_{35}$ Council on the supply and quality of engineering graduates, concluded that curriculum development required a top-down, systematic and holistic approach founded in consideration of specific objectives and graduate attributes (King, 2008). Despite such findings, actual changes to engineering higher education,

40 while laudable, have largely been at the grass roots level, ad hoc and isolated. Furthermore, there is a lack of detail and clarity regarding the content type, program location or the methodology required to develop curricula to best achieve these aims.

In addressing this absence of systemic approach to rapid 45 curriculum renewal, Desha and Hargroves have developed a model as shown in Figure 1, building on curriculum renewal theory, their teaching and learning experiences over the last decade and with peer review by more than 70 engineering academics internationally.

50 This model outlines as a six step process that includes awareness raising and developing a common understanding amongst staff, identifying graduate attributes, auditing and mapping each program against graduate attributes, embarking on strategic content development and renewal, bridging and outreach with ${ }_{55}$ industry and education, and integrating curriculum with campus and community opportunities. Whilst specific examples of graduate attribute development and program mapping are actually quite rare in the literature, the other elements of the model form a well-established and validated framework for curriculum renewal 60 for sustainability (Mulder, 2006).

Figure 1. Desha and Hargroves Deliberative and Dynamic Model for Curriculum Renewal (Desha and Hargroves, 2011)

65

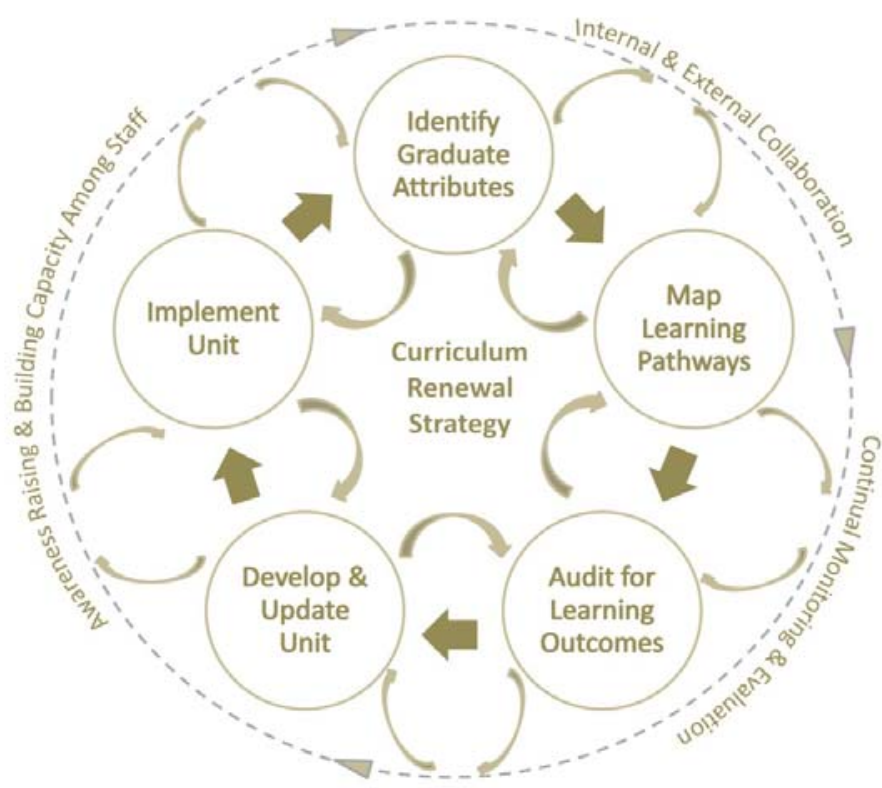

\section{A Case Study of James Cook University's Chemical Engineering Program}

JCU Engineering is a small and highly integrated School with 25 staff offering a 4-year bachelor engineering degree comprising 32 70 subjects (15 discipline, 17 multiple disciplines), with four discipline-major options (chemical, mechanical, civil and electrical engineering). The School's intake comprises approximately 500 - 600 students at any one time, of which approximately 60-80 study chemical engineering. Subsequently, 75 the school operates within a lean operating budget and a staff cohort with significant workload allocations and with directives to optimise delivery of content. In 2009, senior management concluded a need to renew the current degree program over the next five-year accreditation cycle, committing to a period of rapid 80 curriculum renewal, which they termed curriculum refresh (Australian Department of Education, Employment and Workplace Relations, 2009). The overarching aims of the 
curriculum refresh process were to realign undergraduate curricula across the entire university in order to reflect the universities strategic priority areas: sustainability, the tropics and indigenous culture.

${ }_{5}$ Within the context described in the previous sections, the Chemical Engineering department at James Cook University approached the model's authors in 2009 to collaborate in the application and development of the renewal model within its undergraduate chemical engineering program. In contrast to

10 beginning with unit redevelopment, the JCU researchers were interested in an applied research project that took a whole of curriculum approach to embedding sustainability within the curriculum. Subsequently the research team hypothesised that this model could provide a robust framework for comprehensively

15 embedding sustainability concepts across all year levels of a largely traditional chemical engineering degree program. The collaboration secured a component of a federal 'curriculum refresh' grant (Australian Department of Education, Employment and Workplace Relations, 2009) and the authors of this paper 20 undertook a multi-staged mixed-method grounded enquiry project to pilot the model as a framework with which to undertake rapid curriculum renewal. The first few stages involved all four engineering sub-disciplines, after which the chemical engineering school was piloted for the remainder of the model 25 implementation.

The method and results of each aspect of the project are summarised in the following sections, together with insights into new knowledge related to the model's functionality.

\subsection{Awareness Raising and Collaboration}

${ }_{30}$ In the JCU case study, the elements of awareness raising and industry outreach were combined, through a seminar forum advertised both internally (amongst academic staff) and externally (local industries). The general remit of the forum was for local industries to show examples of sustainable engineering 35 practice and to discuss how professional engineers use sustainability within their industry. An expert panel question and answer session was also held to discuss emergent issues for the curriculum, and to brainstorm the types of skills graduates needed in this area.

${ }_{40}$ One of the criticisms of the forum was that its content was too general to inform academics teaching within their specific discipline specialities. To target this gap, the project used some of the research funds to allocate 10 small professional development grants (AUD \$1,000 each) that targeted academic staff aligning

45 their research and teaching focus with sustainability. The grant selection process involved academics describing how the professional development would be used to facilitate the development of curriculum content and was directly aligned to course specific outcomes, such as the development of lecture 50 notes.

Industry outreach also involved discussing the graduate attributes (described in the following section) with a cross-section of local mid-level professional engineers in order to garner their input into attribute selection and graduate qualities. Teaching graduate 55 understanding and skills in sustainability was encouraged by industry. However industry participants did not consider themselves to be in a position to inform higher education on the specific characteristics and skills required. The research team concluded that the JCU chemical engineering school is well 60 placed to demonstrate leadership in this area and to inform and shape future graduates, and via its graduates contribute to shifting the chemical engineering industry to a position of improved sustainability practices.

\subsection{Identifying Graduate Attributes}

${ }_{65}$ Following confirmation of the university's curriculum renewal strategy with university senior management and the awareness raising forum, the project team undertook a series of four discipline-specific workshops, facilitated by The Natural Edge Project, with staff from chemical, mechanical, electrical, and 70 civil engineering. Workshops were well-supported and involved 70 percent of total engineering academic and teaching staff. The high level of participation of teaching staff has a number of benefits. These include a sense of ownership of the project outcomes, potential to align project outcomes with individual 75 teaching strengths, and the ability to shape attributes so that they reflect the unique teaching and research environment at JCU.

During the workshops, participants were first introduced to the curriculum refresh project and then informed about the importance of using a formalised methodology for development 80 of curricula. Educational methods to define generic graduate attributes using words such as appreciation, ability, awareness and competency where described as context for the workshops. These attributes, after modifications described in the following section, would eventually become the framework on which new 85 curriculum would be designed. Using the workshop approach developed by the Desha and Hargroves, participants were asked to brainstorm a list of 15 to 20 sustainability attributes in their discipline. Responses were prompted by the following question: "What do you think the key sustainability attributes for ${ }_{90}$ Chemical/Mechanical/Civil/Electrical Engineering graduates will be over the next 5 years?"

\section{Discipline-Specific Attributes}

The participants first prioritised the attributes within their ${ }_{95}$ discipline to the five most critical or essential attributes, and then jointly determined the extent to which attributes were currently covered in the existing curriculum (low/medium/high). An example of the prioritised attributes emerging from the chemical engineering workshop is presented in Table 4. 
Table 4. An example of prioritised attributes emerging from the chemical engineering graduate competency workshop

\begin{tabular}{|l|l|}
\hline Attribute description & Current course coverage \\
\hline Appreciation of energy efficiency/sustainable energy generation & low \\
\hline An ability to quantify short and long term impacts on society/ environment & low \\
\hline An awareness of the complexity of systems, risk, uncertainty, solutions and optimisation & low/medium \\
\hline Competency in systems design/ analysis & Medium \\
\hline Appreciation of the urgency of environmental issues and knowing their place in society & medium/high \\
\hline
\end{tabular}

\section{${ }_{5}$ Multi-disciplinary Attributes}

The research team realised that in order to facilitate the identification, design, and delivery of sustainability curriculum within a highly integrated program in the most efficient way, discipline specific attributes also needed to be examined in terms 10 of their commonalities. This is particularly important as a key characteristic demonstrating a student's understanding of sustainability is the ability to cross discipline boundaries and broaden their perspective.

Hence, as an extension of the original model methodology, 15 during the workshop participants were also asked to identify areas of specialisation or discipline strengths that may be beneficial to other disciplines in the development of graduate sustainability attributes. Following the workshop, all four discipline (Chem./Mech./Civil/Elect.) attribute lists were then 20 distilled into a series of five common multi-disciplinary attributes, as shown in Table 5. These were grouped under the broad university-wide JCU graduate attribute: Awareness of sustainability to ensure that the project aligned with the overarching university curriculum renewal strategy. It is also

25 worth noting that the use of the term "discipline" within the attribute statements provides for differentiation between the different majors.

Table 5. Engineering graduate attribute list for the JCU 30 university-level attribute "Awareness of Sustainability"

\begin{tabular}{|l|l|}
\hline Graduate attribute description & $\begin{array}{l}\text { Capability } \\
\text { keyword }\end{array}$ \\
\hline $\begin{array}{l}\text { Knowledge of sustainability including definitions, } \\
\text { discipline context, relevance and importance. }\end{array}$ & Knowledge \\
\hline $\begin{array}{l}\text { Discipline specific exposure to sustainability } \\
\text { applications including examples of sustainable } \\
\text { practice and design. }\end{array}$ & Applications \\
\hline $\begin{array}{l}\text { Ability to conceptualise complex systems and theil } \\
\text { interaction across ecological, social and } \\
\text { environmental dimensions. }\end{array}$ & Systems \\
\hline $\begin{array}{l}\text { Ability to use tools to quantify sustainability of } \\
\text { products, processes and designs. }\end{array}$ & Quantify \\
\hline $\begin{array}{l}\text { Ability to optimise engineering designs to trade off } \\
\text { across the three dimensions of sustainability } \\
\text { (environment, equity, economy) }\end{array}$ & Optimise \\
\hline
\end{tabular}

Characteristics of the graduate attribute list include that the 45 attributes are scaffolded such that knowledge will typically precede applications and systems. Furthermore, systems attributes will typically precede quantifying and optimising attributes. The list is also generic enough that discipline specific examples of sustainable practice (such as those that would be presented under 50 the applications attribute) are easily accommodated. An example of this would include applications of green chemistry within chemical engineering and applications of carbon reduction techniques in concrete formulation within civil engineering. The optimise attribute is considered to be a higher level capability that 55 may not necessarily be achieved by all undergraduate engineering students. As such it has been termed a 'stretch target', suitable for developing over a longer timeframe or address within postgraduate courses.

This list was compared to lists generated by accreditation bodies 60 such as EA and IChemE and a high degree of qualitative alignment was obtained, which is important for professionally accredited engineering programs.

\subsection{Mapping Learning Pathways}

It is common practice in addressing emerging knowledge ${ }_{65}$ domains to 'bolt on' a new subject or two. However, there is a strong evidence that suggests this trivialises pervasive issues such as ethics, sustainability, and project management (Mulder et al., 2011). The alternative approach, used as the basis for this project, is to integrate the development of these themes across the 70 curriculum, ideally as a scaffolded sequence of developmental experiences, or 'learning outcomes' that can be demonstrated by students on completion of the subjects. It is common that academics are familiar with where students need to get to in terms of learning objectives or specific skills and abilities, but are 75 unfamiliar with the precise pathways required to achieve this endpoint. Before individual subjects can be renewed, the learning pathways for the attributes need to be identified, through a sequential map of subjects associated with developing the attributes. Then, for each subject, learning outcomes can be 80 generated that meet the attribute development requirements.

Building on the model directions, the project team formulated a workshop-based mapping process to enhance multidisciplinary approaches to learning, minimise content overlap, scaffold learning pathways and broadly identify required content. In the ${ }_{85}$ absence of specific expertise in program-wide curriculum design for sustainability and in the interests of parsimony, a simple mapping process was used. Essentially, 'mapping' refers to 
identifying (as much as possible) specific subject content for specific subjects in each year of study, noting that the learning pathway for sustainability knowledge and skills needs to be carefully sequenced. For example, mapping constraints include 5 an understanding that early year subjects would be unlikely to contain quantify or optimise related content and latter subjects would be unlikely to contain knowledge or applications related content. With this in mind, the process was designed to be completed without the need to identify precise subject content,

10 and without needing to know specific budgetary or staffing constraints.

As a starting point for mapping, a workshop including all discipline teaching staff, industry contract teachers and senior program managers broadly identified a subset of subjects (in the

15 chemical engineering case 12 subjects were selected: 7 multidisciplinary and 5 discipline specific) with suitable themes that could potentially accommodate new or modified content. For example, a second-year, multidisciplinary subject "Thermofluid mechanics" was identified as a subject that might be suitable for

20 embedding examples of energy efficiency. A third-level, multidisciplinary subject "Materials science and engineering" was identified as a subject that might be suitable for embedding concepts such as embedded energy. A third level chemical engineering subject "Reaction Engineering" was identified as a 25 subject that suitable for embedding green chemistry concepts and examples.

\begin{tabular}{|l|l|l|l|l|l|l|l|l|l|l|l|l|l|}
\hline & \multicolumn{3}{c}{ Level 1 subjects } & \multicolumn{3}{c|}{ Level 2 subjects } & \multicolumn{3}{c|}{ Level 3 subjects } & \multicolumn{3}{c|}{ Level 4 subjects } \\
\hline attribute & s1001 & s1002 & s1003 & s2001 & s2002 & s2003 & s3001 & s3002 & s3003 & s4001 & s4002 & s4003 \\
\hline knowledge & & & & & & & & & & & & \\
\hline application & & & & & & & & & & & & & \\
\hline systems & & & & & & & & & & & & \\
\hline quantify & & & & & & & & & & & & & \\
\hline optimise & & & & & & & & & & & & \\
\hline
\end{tabular}

Once the subset of subjects were selected, these were placed in sequential order (by year) across the top of the map (i.e. map columns). The attributes/capabilities identified in the prior stage 30 of the project (knowledge, applications, systems, quantify, optimise) were then placed as rows across the map. Considering the JCU context which varied among the four disciplines, the research team identified two complementary approaches which were used to populate the map.

${ }_{35}$ Content-Generic Approach

In the first approach the mapping is driven by the attributes and is termed a "bottom up" approach and is a useful approach when knowledge of the precise content is not yet known. In this approach the attributes (for example from Table 5) are scaffolded 40 to the four year levels. Careful consideration of the subject material and type of content delivered in each selected subject should be made and appropriate choice of workshop participants is important in this regard. This results in a generic map illustrated in Figure 2. In this map a subset of 3 subjects per year 45 are used to support attribute deployment. The number of subjects chosen is dependent upon local conditions and would have to be decided by the academic leadership. The sequencing of attribute development may also overlap and would be dependent on the types of subjects chosen and their sequencing within an 50 institution's program.

It is worth noting that in this example mapping, the development of the "optimisation" attribute is not completed, and a 5th year of study would be required to achieve this attribute. This may be either a through a Masters program or a 5th year of undergraduate 55 study, such as would be undertaken by students in the "Melbourne model". Each shaded entry in the map then requires the specific set of skills to be attained, specific lecture content to be delivered or specific examples to be illustrated to students. At this stage of the mapping process it is often possible for 60 academics to identify specific content that is suitable for the early years of the program. However, specific content requirements in the middle and latter years of the program can be more difficult to identify. This content gap is targeted using the second approach to mapping described below.

65

Figure 2. Generic curriculum map for embedding sustainability attributes into 4 year engineering programs.

\section{Content-Specific Approach}

In the content-specific approach specific topic areas to be covered by students are integrated into the program map. Specific graduate knowledge and skills are identified throughout the year 90 levels of the degree, providing an immediate indication of the learning outcome statements. This is referred to by the authors as a "top-down" approach, and is driven through the identification of capstone experiences.

In the JCU case study, for chemical engineering the key capstone 95 experiences identified for inclusion comprised the use of life cycle assessment tools or undertaking a life cycle assessment in a student's final year chemical engineering design. Academics and teaching staff recognised that this experience should involve an understanding of systems and interactions between communities, 100 environment and technological processes. Students may be asked to quantify the economics, environmental burdens and social benefits of their process. There is potential for students to optimise across these domains through good design and appropriate technology and chemistry selection. Aligning the 
capstone experience with sustainability attributes staff recognise that the capstone experience demonstrates that systems, quantify and optimise attributes are required in this subject. The challenge then is to back track through the map to earlier years in order to 5 determine within the map the where necessary development skills would be attained. In addition, key words such as "water" and "energy" obtained from the IChemE roadmap were used to provide extra specificity in cell entries. An example of attribute development across the first two years of the chemical ${ }_{10}$ engineering program at JCU is shown in Figure 3. It is worth noting that the map is not fixed but is used as a guide to inform content development and as a mechanism to describe attribute development (within accreditation submissions, for example). As mapping progresses from one discipline to the next, the mappings 15 will become more complex with their multidisciplinary overlaps. A feedback and adjustment process would need to be included to account for these complexities when extending the methodology to other disciplines. Where gaps in the map were identified, potentially as a result of a lack of the required in-depth 20 knowledge by workshop participants, the research team utilised project funds to hire a research assistant to identify content.

Figure 3. Curriculum map for the 1st and 2nd year of the chemical engineering program.

\subsection{Developing and updating courses}

30 The first two authors of this paper have to this date conducted a selection of course-by-course reviews of the chemical engineering curriculum, starting at 1st year level and progressing to final year courses. Course outlines and information obtained through a short interviews with subject convenors/lecturers where 35 used to identify resource requirements and determine aligned professional development opportunities.

It is noteworthy that the subsequent process yielded a number of insights

- The subject convenors were generally amenable to incorporating the desired changes within their subjects, once the opportunity for renewal had been formally identified,

- A number of subjects were already addressing the desired learning outcomes but they had not been clearly articulated in subject outlines.

- Subject convenors were most open to and supportive of curriculum renewal when teaching and curriculum demands were aligned with research interests and coupled to professional development opportunities.

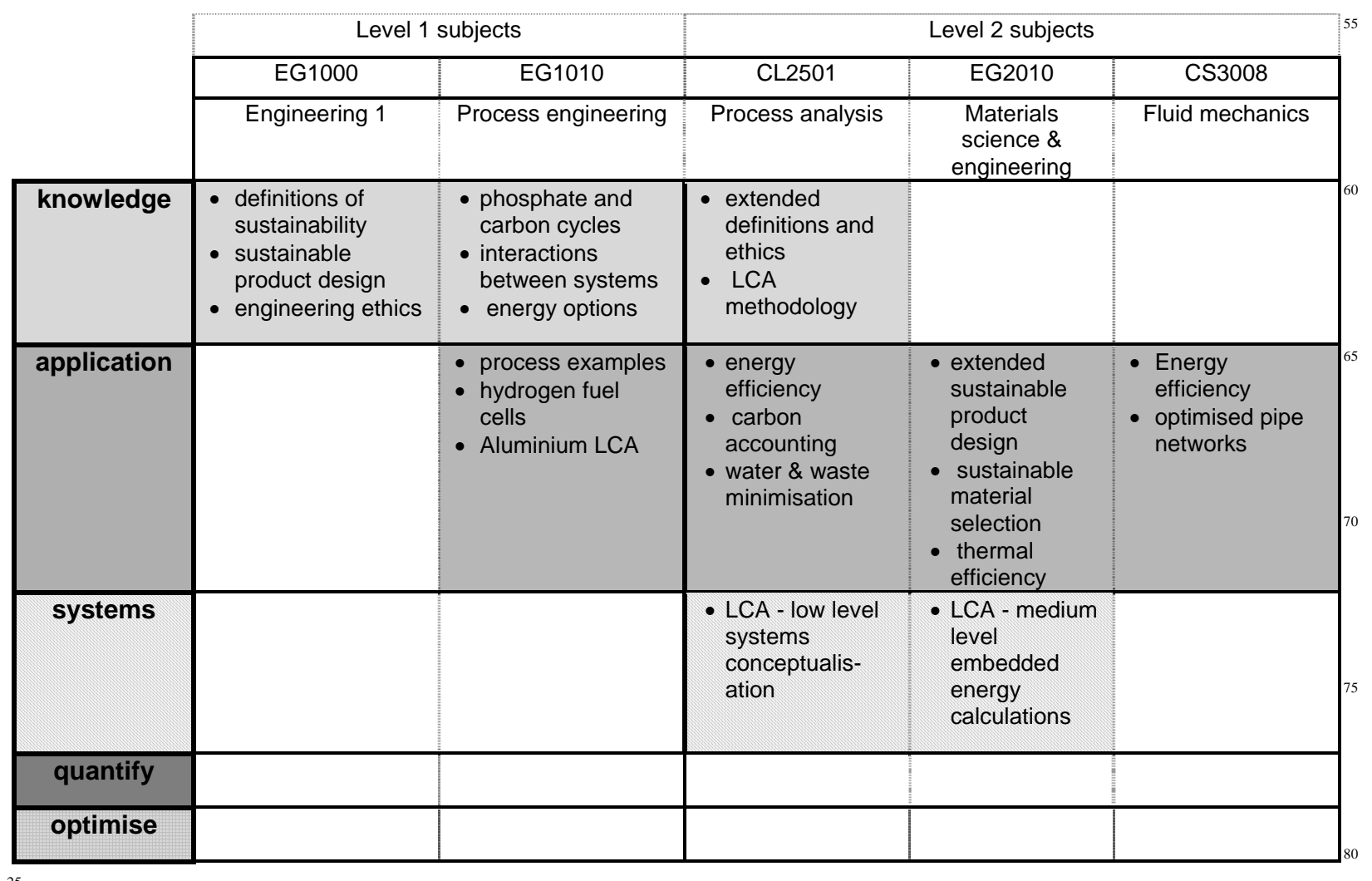


Following the identification and mapping steps, defining the specific content requirements for subjects in a program is an iterative process which requires a high level of understanding and knowledge of the application of sustainability attributes to 5 engineering problems. At this point in the project, the research team sought feedback from staff about the level of awareness of resources for course renewal. It quickly became apparent that most staff found online searching for resources overwhelming and difficult to align with individual teaching approaches.

10 Furthermore, there are very few examples of discipline core textbooks that include sustainability as an emphasis or provide examples which emphasise sustainable practice. For example, in the JCU case study, content in a 2nd year course (Process Analysis - covering energy balances and design) was obtained 15 through discussions and exchanges with external life cycle application experts who had developed and written their own materials, based predominately on postgraduate research projects. This demonstrates the emerging nature of sustainability skills and knowledge as well as the challenge and resource intensive 20 demands involved in sourcing teaching content. Furthermore, learning pathways required to enable students to confidently undertake life cycle analysis (LCA) and triple bottom line (TBL) by graduation are still being established in this emerging field.

From a practical perspective it is worth noting that academic 25 workloads are significant. Sourcing and delivering new content places an additional strain on academics that is commonly seen as a burden that reduces their ability to undertake research. As such, alignment of content as much as possible with academic research specialities is of great benefit both to academic 30 motivation and ultimately facilitates better learning and teaching outcomes. However, research alignment cannot always be achieved and allowing a long lead in time and positive reinforcement of changes academics make is helpful. Mapping is also helpful in this regard as the timing and sequencing of course 35 changes can be deferred for latter subjects (such as 3rd and 4th year level subjects). Which allows motivated or research aligned academics to be brought in to modify early subjects (1st and 2nd year level subjects) and act as exemplars of best practice.

\subsection{Conclusions and Next Steps}

40 The research team has successfully defined a list graduate attributes which will ultimately lead to students graduating from JCU-chemical engineering being capable of demonstrating an "awareness of sustainability". Awareness raising, professional development, resource support and senior management support 45 and leadership are seen as integral to successful curriculum renewal. Mapping tools were developed as part of the case study to facilitate both content identification and content location within the undergraduate chemical engineering program. To date, of the 12 subjects selected only 9 subjects have been updated 50 including 6 discipline and 3 multi-discipline subjects.

Enhanced professional development opportunities and alignment of staff research strengths toward a common "sustainability" ethos have led to new relationships being developed with community and corporate stakeholders (such as Townsville City

${ }_{55}$ Council and Ergon Energy) as well as cross institutional collaboration. Furthermore, integrative projects with campus entities such as the JCU Tropeco -sustainability group and the
JCU - School of Education will continue to expand the level of interaction between engineering staff and external stakeholders. 60 These are all seen as welcome additions that ultimately enhance the long term viability of the curriculum refresh process.

This project was an important milestone in the development of the deliberative and dynamic model for curriculum renewal, permitting a whole of method trial and evaluation through the ${ }_{65}$ JCU cases study. The JCU case study highlighted the identification, mapping and course updating process as effective means to determine and design the development of chemical engineering attributes as well as defining appropriately scaffolded learning outcome statements for the various subjects to be 70 renewed, and to elucidate the content required within each of the subjects. The JCU experience also reinforced the model's depiction of the iterative nature of all steps, in particular awareness raising and monitoring and evaluation.

In conclusion, RCR and attribute mapping provides a sound and 75 repeatable methodology that has been successfully used to identify and embed sustainability content within the JCU chemical engineering program. Strong participation rates in workshops meant that the derived sustainability attributes were felt to be "owned" by staff and the attributes were condensed 80 sufficiently to allow the required flexibility in content development. This is important as freedom and independence are highly valued characteristics of academic teaching staff. Mapping was found to be an essential and valuable tool to visualise whole of program linkages and to facilitate the long term timing of 85 content and curriculum renewal.

The next steps for the JCU chemical engineering program includes undertaking subject renewal for the whole curriculum renewal map. This will be rolled out over the next few years, implementing the changes and monitoring and evaluating for 90 further improvement. Forums on sustainability, local industry engagement and integrative projects with campus entities will continue to be undertaken. In the larger context of JCU, there are also plans to bring the other three discipline areas to the same stage of curriculum renewal as chemical engineering (i.e. through ${ }_{95}$ defining learning outcomes and updating the existing curriculum). With regard to the model for curriculum renewal, following this whole of curriculum experience at JCU, the model will also need to be piloted in a similar process elsewhere to further improve understanding of the elements of curriculum 100 renewal and their interactions.

Given the resource gaps and the need to facilitate sharing and widespread dissemination of the developed curriculum content, it is also concluded that an online tool for engagement and resource sharing targeted at engineering education would be beneficial 105 within the engineering academic community. Portal-based access to teaching resources across the sustainability spectrum would provide a basis for sharing knowledge, content, and experience between all academics and students of sustainable engineering practices, as well as creating a community of practice where 110 industry could participate in the development of knowledge about sustainability. 


\section{Notes and references}

${ }^{a}$ School of Engineering and Physical Sciences, James Cook University, Townsville, Australia. Fax: 61747751184 Tel:61 74781 4153;Email: madoc.sheehan@jcu.edu.au

$5{ }^{b}$ Faculty of Built Environment and Engineering, Queensland University of Technology, Brisbane, Australia; The Natural Edge Project Research Group; Email: cheryl.desha@qut.edu.au

Australian Dept. Education, Employment and Workplace Relations. 10 (2009-2011) Diversity \& structural adjustment fund, JCU curriculum resfresh project - Australia's University of the Tropics.

Azapagic, A., Perdan, S., and Shallcross, D. (2005), How much do engineering students know about sustainable development?, European J. Engineering Education, 30(1), 1-19.

15 Byrne, E., Desha, C., Fitzpatrick, J. \& Hargroves, K., (2010). Engineering Education For Sustainable Development: A Review Of International Progress, in Proceedings of the 3rd International Symposium for Engineering Education, University College Cork, Ireland.

Byrne, E.P and Fitzpatrick, J.J., (2009) Chemical engineering in an 20 unsustainable world: obligations and opportunities, Education for Chemical Engineers, 4, 51-67.

Davidson, C.I. H. Matthews, S. Hendrickson, C.T. Bridges, M.W Allenby, B.R. Crittenden, J.C. Chen, Y. Williams, E. Allen, D.T. Murphy, C.F. and Austin, S., (2007) Adding sustainability to the

25 engineer's toolbox: a challenge for engineering educators, Environ Sci. Technol., 41(14) 4847-4850.

Desha, C. and Hargroves, K., (2009) Addressing the time lag dilemma in curriculum renewal towards engineering education for sustainable development, Int'l J. Sustainability in Higher Education, 10(2), 184-

$30 \quad 199$

Desha, C. and Hargroves, K. (2011) Informing engineering education for sustainable development using a deliberative dynamic model for curriculum renewal in Proceedings of the 2011 Research in Engineering Education Symposium, Madrid, Spain.

35 Engineers Australia accreditation board: Doc P05 Rev.1, (2006) EA national generic competency standards - stage 1 competency standards for professionl engineers.

A Guide for University Departments and Assessors: Based on Learning Outcomes, IChemE.

40 King, R. (2008) Addressing the Supply and Quality of Engineering Graduates for the New Century, Report to the Carrick Institute for Learning and Teaching in Higher Education Ltd, Sydney.

Mulder, K.F. (2006) Engineering curricula in sustainable development. An evaluation of changes at Delft University of Technology, European Journal of Engineering Education, 31(2), 133-144.

Mulder, K., Desha, C. and Hargroves, K. (2011) Sustainable Development as a Meta-Context for Engineering Education, in Proceedings of the $6^{\text {th }}$ Conference on Sustainable Development of Energy, Water and Environment Systems, Dubrovnik, Croatia.

50 The Royal Academy of Engineering (2007). Educating Engineers for the 21st Century, London: The Royal Academy of Engineering.

Smith, M., Hargroves, K. and Desha, C. (2010) Cents and Sustainability Securing Our Common Future by Decoupling Economic Growth from Environmental Pressures, Earthscan, London.

55 Technical Strategy Roadmap: Progress Report (2008), Driving in the right direction, IChemE.

World Commission on Environment and Development (1987) Our Common Future, Oxford University Press, Oxford, p113. 